\title{
Trefoil Factor 1 Suppresses Epithelial-mesenchymal Transition through Inhibition of TGF-beta Signaling in Gastric Cancer Cells
}

\author{
Da-Young Lee, Moon-Young Song, Eun-Hee Kim \\ College of Pharmacy and Institute of Pharmaceutical Sciences, CHA University, Seongnam, Korea
}

\begin{abstract}
Gastric cancer is a malignancy with high incidence and mortality worldwide. In gastric cancer, epithelial-mesenchymal transition (EMT) and metastasis further increase the mortality rate. Trefoil factor 1 (TFF1) has been reported as a protective factor in the gastric mucosa. In this study, TFF1 inhibited the migration and invasive capability of gastric cancer cells. Elevated TFF1 levels induced the expression of E-cadherin, the epithelial marker, and reduced the expression of N-cadherin, vimentin, Snail, Twist, Zinc finger E-box binding homeobox (ZEB) 1 and ZEB2, well-known repressors of E-cadherin expression. In addition, the expression of matrix metalloproteinase (MMP)-2, MMP-7 and MMP-9, which are major markers of cancer metastasis, was suppressed by TFF1. Upregulation of TFF1 inhibited TGF- $\beta$, a major signaling for EMT induction, and the phosphorylation of Smad2/3 activated by TGF- $\beta$ in AGS cells. In conclusion, TFF1 inhibits EMT through suppression of TGF- $\beta$ signaling in AGS cells, which might be used in therapeutic strategies for reducing metastatic potential and invasiveness of these cells.
\end{abstract}

Key Words Gastric cancer, Trefoil factor 1, Epithelial-mesenchymal transition, Transforming growth factor beta

\section{INTRODUCTION}

Although the incidence and mortality rates of gastric cancer have decreased in recent years, gastric cancer is still prevalent worldwide [1]. In particular, gastric cancer has been reported as the second most common malignancy in Korea [2]. Although resection is a method of treating gastric cancer, it is often difficult to detect gastric cancer in an early stage because there are no symptoms. In the case of more advanced gastric cancer, even if detected, the effect of resection is insignificant, and the prognosis is poor [3]. The major causes of gastric cancer are Helicobacter pylori, gastric ulcer and Epstein-Barr virus, and in addition, genetic and environmental factors are also known to affect it [1]. However, recent studies have reported that the occurrence of gastric cancer is closely related to epithelial-mesenchymal transition (EMT) [3]. EMT promotes metastasis and recurrence of gastric cancer. EMT is the process by which epithelial cells are transformed into mesenchymal cells, and often accompanied by changes such as loss of cellular adhesion and polarity, and gain of mobility and invasive features [3]. Therefore, it is important to control
EMT in order to suppress the gastric tumorigenesis.

In gastric cancer, EMT has been known to be regulated by various growth factors and differentiation factors such as TGF- $\beta$, Wnt or Notch [4]. Among them, TGF- $\beta$ has been attracting attention as a major signaling molecule [5]. Treatment with TGF- $\beta$ has been reported to change epithelial cell morphology, reduce the expression of epithelial markers, and increase the expression of mesenchymal markers [6]. In addition, the TGF- $\beta$ signaling pathway is well known as a major regulator of metastasis through EMT activation in various forms of cancer [5]. Indeed, upregulated TGF- $\beta$ signaling leads to lymph node metastasis, reduced survival, and poor prognosis in gastric cancer [6]. Therefore, inhibition of EMT through regulation of the TGF- $\beta$ signaling pathway is an effective way to inhibit the onset and progression of gastric cancer.

Trefoil factor (TFF) 1, which has a trefoil domain, is synthesized and released by mucous secreting epithelial cells lining the gastrointestinal tract [7]. In the stomach, TFF1 is expressed with mucins and helps maintain mucosal integrity [8]. TFF1 has been studied in various malignancies

Received May 21, 2021, Revised June 19, 2021, Accepted June 21, 2021

Correspondence to Eun-Hee Kim, E-mail: ehkim@cha.ac.kr, https://orcid.org/0000-0002-8523-0440

Check for updates 
such as breast cancer, pancreatic cancer, colon cancer, and gastric cancer [9]. In particular, it has been reported that TFF1-knockout mice exhibit abnormal gastric mucosa and may cause gastric cancer [10]. In addition, the treatment with recombinant TFF1 has been reported to inhibit cell proliferation in vitro and decrease the expression of TFF1 in human gastric adenoma and carcinomas [11,12]. Moreover, recent studies have shown that TFF1 inhibits EMT in the epithelium of pancreatic cancer [13]. It has also been shown that TFF1 inhibits inflammation caused by Helicobacter pylori during gastric cancer progression [14]. Although there are many reports on the gastrointestinal protective effect of TFF1, studies on the association between TFF1 and EMT/metastasis are still insufficient. In particular, the relationship between TFF1 and TGF- $\beta$, which plays an important role in EMT progression, has not yet been fully understood. In the present study, we found that TFF1 inhibits EMT activation through regulation of TGF- $\beta$ signaling pathways, thereby suppressing the metastatic potential in gastric cancer AGS cells.

\section{MATERIALS AND METHODS}

\section{Cloning and expression vectors}

Human TFF1 cDNA was amplified from human gastric cancer KATOIII cells using the specific primers: Hindlll tailed (5'ATG CAA GCT TAT GGC CAC CAT GGA GAA CAA-3') and BamHI tailed (5'-ATG CGG ATC CGC AAA TTC ACA CTC CTC-3'). The PCR product was ligated to HindIII and BamHI digested vector pEGFP-N1 using Quick Ligation Kit (New England Biolabs, Ipswich, MA, USA) following the manufacturer's instruction. Then, the products were transformed into competent Escherichia coli $\mathrm{DH} 5 \alpha$ cells and inoculated into Luria-Bertani culture media (BD Bioscience, Franklin Lakes, NJ, USA) containing kanamycin (Sigma-Aldrich, St. Louis, MO, USA). The following day, one colony was selected and incubated overnight at $37^{\circ} \mathrm{C}$ in Luria-Bertani media containing kanamycin, DNA was extracted using a Exprep Plasmid SV (GeneAll Biotechnology, Seoul, Korea), and the TFF1 sequence was confirmed.

\section{Cell culture}

Human gastric cancer AGS cells were acquired from the American Type Culture Collection (ATCC, Rockville, MD, USA) and maintained in RPMI 1640 medium supplemented with $10 \%$ FBS and $100 \mathrm{U} / \mathrm{mL}$ penicillin and $100 \mathrm{U} / \mathrm{mL}$ streptomycin at $37^{\circ} \mathrm{C}$ in a humidified $5 \% \mathrm{CO}_{2}$ atmosphere. The cells were plated at an appropriate density according to each experiment.

\section{Wound-healing assay}

AGS cells $\left(1 \times 10^{5}\right)$ were seeded in a 6 -well plate. On the following day, each plate was scraped with a pipette tip and transfected with TFF1. After additional incubation for 24 hours, the progression of wound closure was visualized under the microscope (Eclipse 50i; Nikon Inc., Melville, NY, USA).

\section{Transwell invasion assay}

The invasion assay was conducted using the Transwell system (Corning Inc., Corning, NY, USA). The inside of the transwell plates was treated with $0.1 \%$ gelatin (Sigma-Aldrich). AGS cells were transfected with TFF1, and cells were harvested 6 hours later, $1 \times 10^{4}$ cells were placed in $0.2 \mathrm{~mL}$ serum-free media (transwell top chamber), and $0.5 \mathrm{~mL}$ of media containing $10 \%$ FBS was added to the lower chamber. After 48 hours, the cells were fixed with $95 \%$ ethanol and stained with $0.2 \%$ crystal violet (Sigma-Aldrich) for 30 minutes, washed with phosphate-buffered saline and visualized under a microscope. Data were recorded from three random fields of the lower membrane surface and analyzed in triplicates.

\section{Transient transfection and the luciferase reporter gene assay}

AGS cells were transfected with TFF1, E-cadherin, Smad-binding element (SBE) and CAGA promoter-driven luciferase reported plasmid using Lipofectamine ${ }^{\circledR} 2000$ Transfection Reagent (Invitrogen, Waltham, MA, USA) following the manufacturer's protocols. Cells were lysed 24 hours after transfection, and the luciferase assay was performed according to the manufacturer's protocol (Promega, Madison, WI, USA). Each experiment was performed in triplicates.

\section{Western blot assay}

AGS cells were collected with cell lysis buffer containing protease inhibitor (Roche Applied Science, Mannheim, Germany). The cells were centrifuged for 15 minutes at 13,000 rpm. The concentration of protein was determined using a Pierce BCA Protein assay kit (Thermo Fisher Scientific, Rockford, IL, USA). The protein was loaded $10 \%$ SDS-PAGE and transferred to polyvinylidene fluoride membranes, which were incubated with the primary antibodies (diluted 1:1,000). Then, membranes were washed and incubated with peroxidase-conjugated secondary antibodies. Membranes were rewashed, and then were visualized using an enhanced chemiluminescence system (Thermo Fisher Scientific, Waltham, MA, USA). The primary antibodies used for the Western blot analysis are as follows: E-cadherin from BD Bioscience (Franklin Lakes, NJ, USA). TGF- $\beta$ receptor I (TGF- $\beta$ RI), $\mathrm{N}$-cadherin, vimentin, snail, Twist, Zinc finger E-box binding homeobox (ZEB) 1, ZEB2 (also known as Smad interacting protein 1) , TFF1, matrix metalloproteinase (MMP)-2, MMP-7 and MMP-9 from Santa Cruz Biotechnology (Santa Cruz, CA, USA). p-Smad2, p-Smad3, Smad2/3 and Smad7 from Cell Signaling Technology (Danvers, MA, USA).

\section{RNA isolation and RT-PCR}

Total mRNA was isolated from AGS cells using TRIzol ${ }^{\circledR}$ reagent (Invitrogen, Carlsbad, CA, USA) according to the man- 
ufacturer's protocol. cDNA was synthesized using a SuperScript ${ }^{\circledR}$ II Reverse Transcriptase kit (Invitrogen, Waltham, MA, USA) according to the manufacturer's protocol. The mRNA levels were analyzed by reverse transcription PCR (RT-PCR). The relative quantities of target genes were analyzed from triplicates after normalization by $18 \mathrm{~s}$ rRNA. The cDNA was amplified in sequential reactions: $95^{\circ} \mathrm{C}$ for 1 minute, $58^{\circ} \mathrm{C}$ for 30 seconds, and $72^{\circ} \mathrm{C}$ for 1 minute, for 32 cycles of E-cadherin; $95^{\circ} \mathrm{C}$ for 1 minute, $58^{\circ} \mathrm{C}$ for 30 seconds, and $72^{\circ} \mathrm{C}$ for 1 minute, for 35 cycles of $\mathrm{N}$-cadherin and vimentin; $95^{\circ} \mathrm{C}$ for 1 minute, $58^{\circ} \mathrm{C}$ for 30 seconds, and $72^{\circ} \mathrm{C}$ for 1 minute, for 40 cycles of Twist, ZEB2, MMP-2, MMP-7 and MMP-9; $95^{\circ} \mathrm{C}$ for 1 minute, $60^{\circ} \mathrm{C}$ for 30 seconds, and $72^{\circ} \mathrm{C}$ for 1 minute, for 35 cycles of TFF $1 ; 95^{\circ} \mathrm{C}$ for 1 minute, $60^{\circ} \mathrm{C}$ for 30 seconds, and $72^{\circ} \mathrm{C}$ for 1 minute, for 28 cycles of $18 \mathrm{~s}$ rRNA. The primers used for the RT-PCR reactions are as follows (forwards and reverse, respectively): TFF1, 5'-GCA AAT AAG GGC TGC
TGT TTC-3' and 5'-GAA GCG TGT CTG AGG TGT CC-3'; E-cadherin, 5'-GAA CTG CAA AGC ACC TGT GAG-3' and 5'-TCG ACC GGT GCA ATC TTC AA-3'; N-cadherin, 5'-GAC AAT GCC CCT CAA GTG TT-3' and 5'-CCA TTA AGC CGA GTG ATG GT-3'; vimentin, 5'-GAG AAC TTT GCC GTT GAA GC-3' and 5'-TCC AGC AGC TTC CTG TAG GT-3'; Twist, 5'GGA GTC CGC AGT CTT ACG AG-3' and 5'-TCT GGA GGA CCT GGT AGA GG-3'; ZEB2, 5'-TTC CTG GGC TAC GAC CAT AC-3' and 5'-GCC TTG AGT GCT CGA TAA-3' ; MMP2, 5'-GCG ACA AGA AGT ATG GCT TC-3' and 5'-TGC CAA GGT CAA TGT CAG GA-3'; MMP-7, 5'-GTG GTC ACC TAC AGG ATC GT-3' and 5'-ACC ATC CGT CCA GCG TTC AT3'; MMP-9, 5'-CGC AGA CAT CGT CAT CCA GT-3' and 5'GGA TTG GCC TTG GAA GAT GA-3'; and 18s rRNA, 5'-CCC AAC TTC TTA GAG GGA CAA GT-3' and 5'-TAG TCA AGT TCG ACC GTC TTC TC-3'. Amplification products were analyzed in $1.5 \%$ agarose gel electrophoresis, stained with red
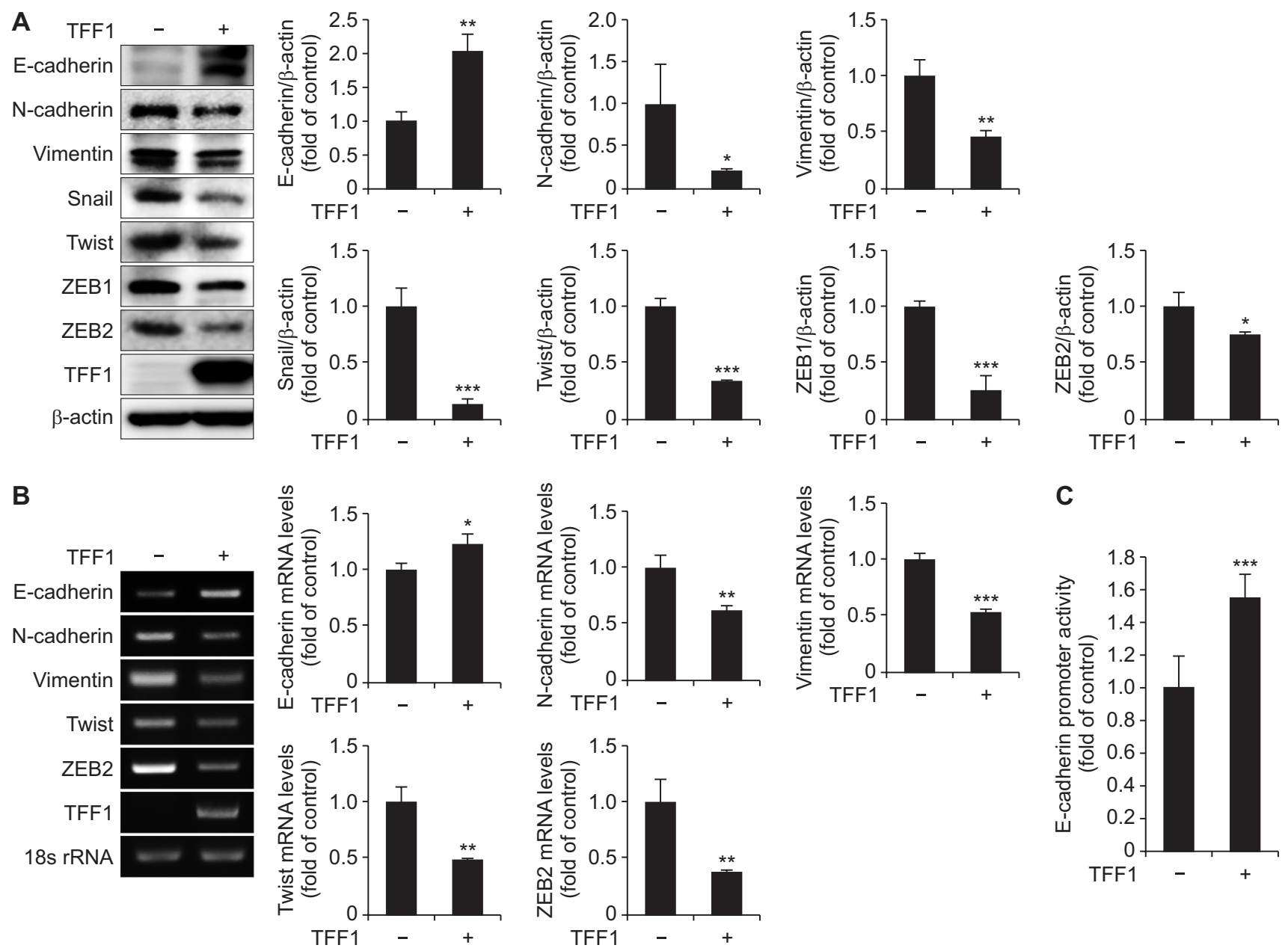

Figure 1. Trefoil factor 1 (TFF1) inhibits epithelial-mesenchymal transition (EMT) in AGS cells. (A) AGS cells were transfected with TFF1 for 24 hours. Western blot analysis was conducted for measuring the expression of E-cadherin, N-cadherin, vimentin, Snail, Twist, ZEB1 and ZEB2. (B) AGS cells were transfected with TFF1 for 24 hours. Reverse transcription PCR (RT-PCR) was conducted to measure the mRNA transcripts of E-cadherin, $\mathrm{N}$-cadherin, vimentin, Twist and ZEB2. (C) AGS cells were transiently transfected with TFF1 and E-cadherin plasmids for 24 hours. Cell extracts were harvested, and the luciferase assay was performed. ZEB, Zinc finger E-box binding homeobox. ${ }^{\star} P<0.05$, ${ }^{\star \star} P<0.01$, and ${ }^{\star \star \star} P<0.001$, significantly different compared with control. 
safe, and photographed under ultraviolet light.

\section{Statistical analysis}

All data are expressed as means \pm SD. Each experiment was performed a minimum of three times. Statistical analysis was performed using one-way ANOVA. Statistical significance was accepted at $P<0.05$.

\section{RESULTS}

\section{TFF1 inhibits EMT in human gastric cancer AGS cells}

TFF1 has been reported to reduce inflammation in the stomach and inhibit gastric cancer progression $[15,16]$. In addition, recent studies have also reported that TFF1 can inhibit EMT [13]. In order to figure out the relevance of TFF1 to EMT, we investigated the expression of EMT markers and E-cadherin promoter activity. As a result, the expression of E-cadherin was significantly increased in TFF1-transfected cells, whereas EMT-inducing factors such as $\mathrm{N}$-cadherin, vimentin, Snail, Twist, ZEB1 and ZEB2 were significantly downregulated (Fig. 1A). In parallel with elevated expression of proteins, the mRNA transcript of EMT markers were also decreased by transfection with TFF1 in gastric cancer AGS cells. As shown in Figure 1B, TFF1 induced the mRNA expression of E-cadherin, whereas TFF1 suppressed that of $\mathrm{N}$-cadherin, vimentin, Twist and ZEB2. In particular, Western blot analysis and
RT-PCR showed significant downregulation of Snail, ZEB1, and ZEB2, which are well known repressors of E-cadherin expression, while the epithelial marker E-cadherin was significantly upregulated. Next, we determined the effects of TFF1 on the promoter activity of E-cadherin. Elevated TFF1 levels enhanced E-cadherin luciferase activity (Fig. 1C).

To verify the role of TFF1 in the regulation of EMT, we performed wound-migration and invasion assays in AGS cells transfected with TFF1. TFF1 transfection for 24 hours reduced the migrative capability of AGS cells (Fig. 2A). To further understand the role of TFF1 in invasiveness and EMT switching, we performed the transwell invasion assay. We found that the invasive property of AGS cells was significantly decreased by transfection with TFF1 (Fig. 2B).

\section{TFF1 inhibits the expression of metastatic markers in human gastric cancer AGS cells}

In cancer cells, the progression of EMT is well known to induce metastasis [17]. We observed that TFF1 inhibited EMT, migration, and invasiveness in AGS cells in Fig. 2. This prompted us to examine the effects of TFF1 on the expression of metastatic markers, MMPs, which degrade extracellular matrix as a prerequisite for cellular invasion and is involved in several types of cancer metastasis. Transfection of AGS cells with TFF1 significantly reduced the expression of MMP2, MMP-7, and MMP-9 (Fig. 3A). and their mRNA transcripts (Fig. 3B).
A

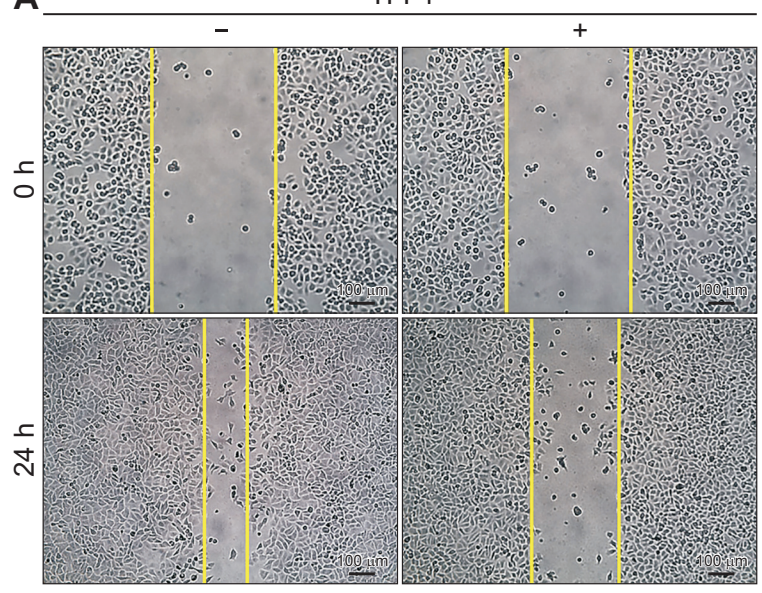

B

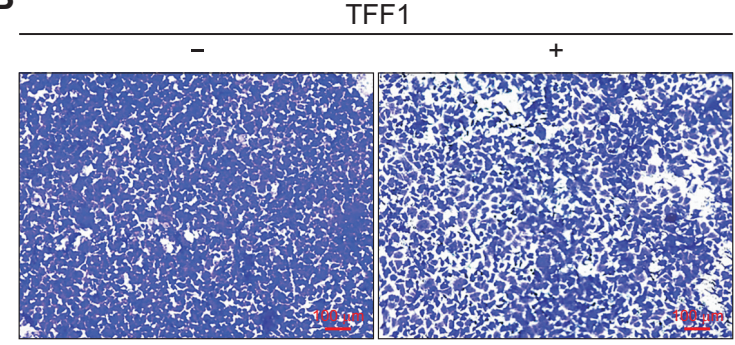

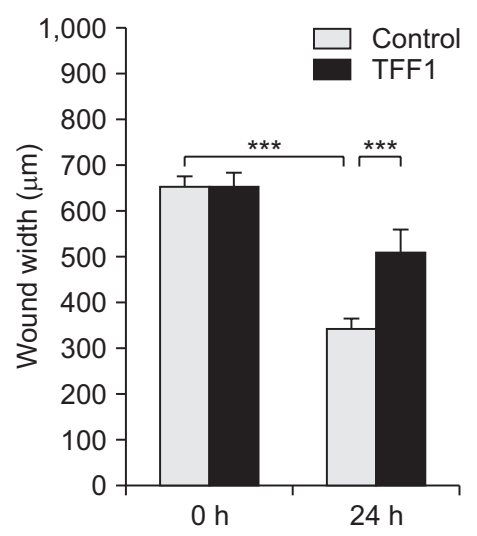

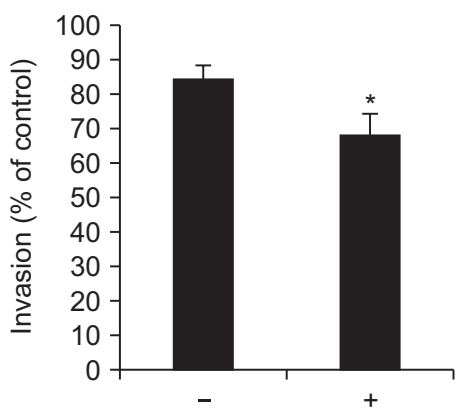

Figure 2. TFF1 suppresses migration and invasion in AGS cells. (A) AGS cells were transfected with TFF1 for 24 hours. After transfection, the cell migration was analyzed for 24 hours in scratched AGS cells. (B) Cell invasion was analyzed after 48 hours post transfection with TFF1 to allow for the permeabilization of the transwell membrane. The membrane was stained with $0.2 \%$ crystal violet. Scale bar, 100 $\mu \mathrm{m}$. TFF1, Trefoil factor $1 .{ }^{*} P<0.05,{ }^{* *} P$ $<0.01$, and ${ }^{* * *} P<0.001$, significantly different compared with control. 

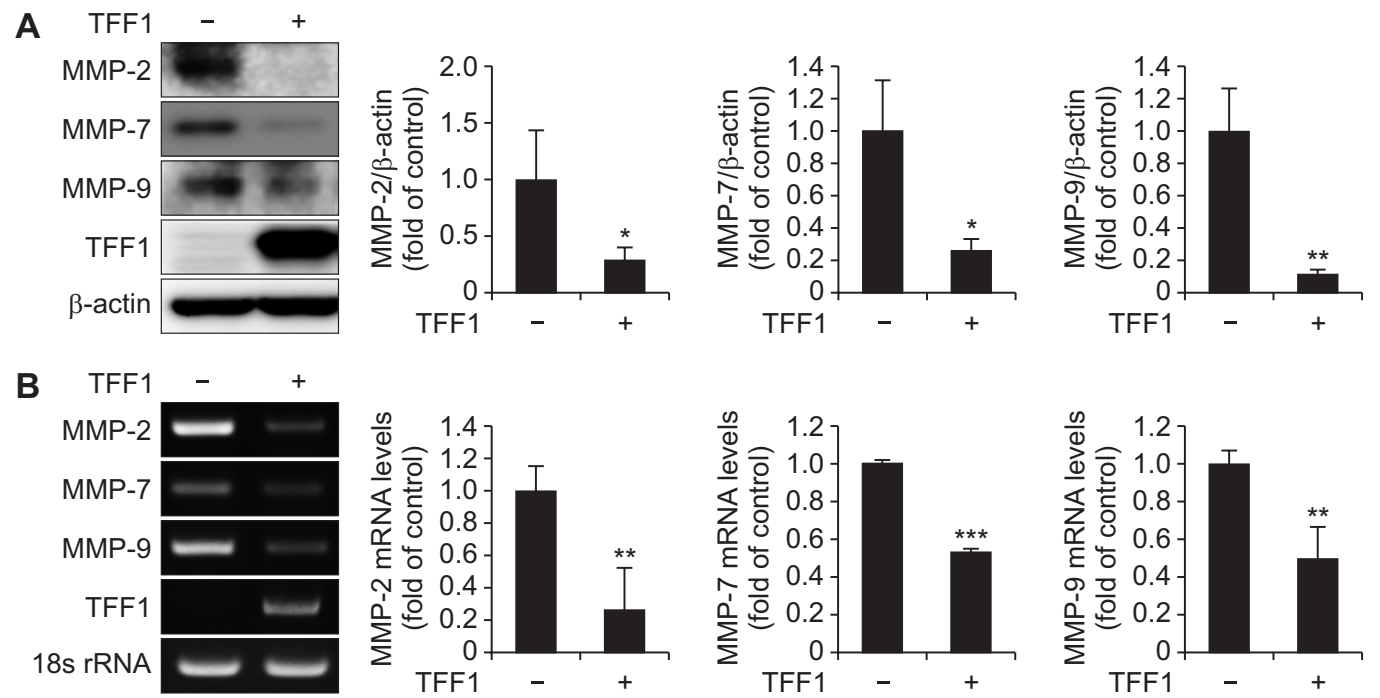

Figure 3. TFF1 inhibits the expression of metastatic markers in AGS cells. (A) AGS cells were transfected with TFF1 for 24 hours. Western blot analysis was conducted for measuring the expression of metalloproteinase (MMP)-2, MMP-7 and MMP-9. (B) AGS cells were transfected with TFF1 for 24 hours. RT-PCR was conducted for measuring the mRNA expression of MMP-2, MMP-7 and MMP-9. TFF1, Trefoil factor 1 . ${ }^{*} P<0.05,{ }^{* *} P<0.01$, and ${ }^{* *} P<0.001$, significantly different compared with control.

\section{TFF1 inhibits the TGF- $\beta$ signaling pathway}

The TGF- $\beta /$ Smad signaling pathway has been reported to play a crucial role in the EMT and to promote metastasis in gastric cancer [18]. Therefore, we measured the activity of Smad-binding element (SBE) and CAGA, known as TGF- $\beta$ responsive motifs, in AGS cells overexpressing TFF1. As a result, upregulation of TFF1 significantly reduced the SBE and CAGA promoter activities in AGS cells transfected with TFF1 (Fig. 4). Next, we measured the protein expression of molecules related to the TGF- $\beta$ signaling pathway by Western blot analysis. Treatment of AGS cells with TGF- $\beta$ significantly induced the expression of TGF- $\beta$ RI, phospho-Smad 2 and phospho-Smad3. However, transfection with TFF1 significantly inhibited the increased levels of TGF- $\beta$ RI, phospho-Smad2 and phospho-Smad3 activated by TGF- $\beta$ treatment in AGS cells (Fig. 5). On the other hand, Smad7, which was reduced by TGF- $\beta$ treatment, was recovered in TFF1-transfected AGS cells. Taken together, these findings indicate that TFF1 has the inhibitory effect on the TGF- $\beta / S m a d$ signaling pathway in gastric cancer AGS cells.

\section{DISCUSSION}

Gastric cancer is one of the most common malignancies in the world [1]. Invasion and metastasis are the main causes of death from gastric cancer [19]. There are multiple lines of evidence supporting the importance of EMT, which transforms epithelial cells into mesenchymal forms [20]. EMT appears in various lesion processes such as inflammation and fibrosis, mainly characterized by a decrease in epithelial markers with an concomitant increase in mesenchymal markers [21]. During EMT, E-cadherin, a cell adhesion protein, is converted
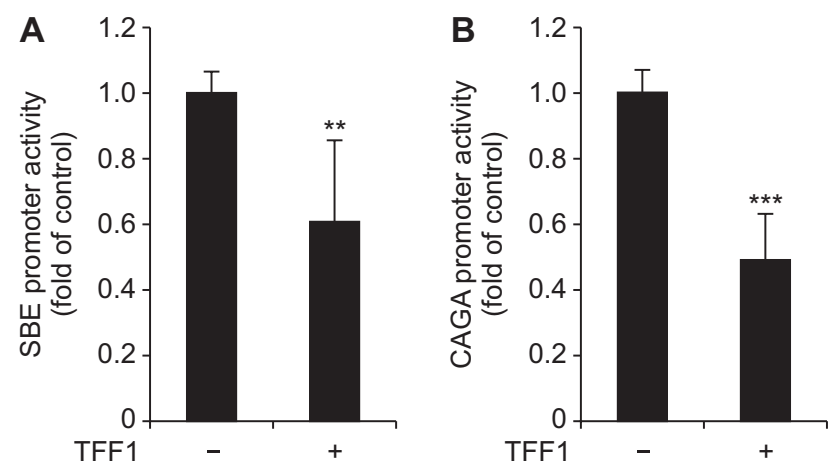

Figure 4. TFF1 inhibits TGF- $\beta$ promoter activity in AGS cells. The activity of Smad binding element (SBE) (A) or CAGA luciferase reporter (B) was measured in AGS cells. AGS cells were transiently transfected with TFF1 and SBE (A) or CAGA plasmids (B) for 24 hours. Cell extracts were harvested, and luciferase assays were performed. TFF1, Trefoil factor $1 .{ }^{* *} P<0.01$ and ${ }^{* * *} P<0.001$, significantly different compared with control.

to $\mathrm{N}$-cadherin which is accompanied by the increase in the levels of vimentin [20]. The decrease in E-cadherin is caused by various major EMT-induced transcription factors including Snail1, Snail2, Twist, ZEB1, ZEB2, forkhead box C2, E47, and so on. These transcription factors influence one another and have been known to be downstream of TGF- $\beta$, Wnt, Notch, signal transducer and activator of transcription-3, epidermal growth factor and NF-кB $[22,23]$. In this study, we investigated the effect of TFF1 on the expression of major EMT markers and EMT-related promoter activity.

TGF- $\beta$ has been recognized as a major effector promoting EMT and metastasis [5,7]. In cancer cells, the activity of TGF- $\beta$ promotes EMT and imparts invasion to cells [5]. The 

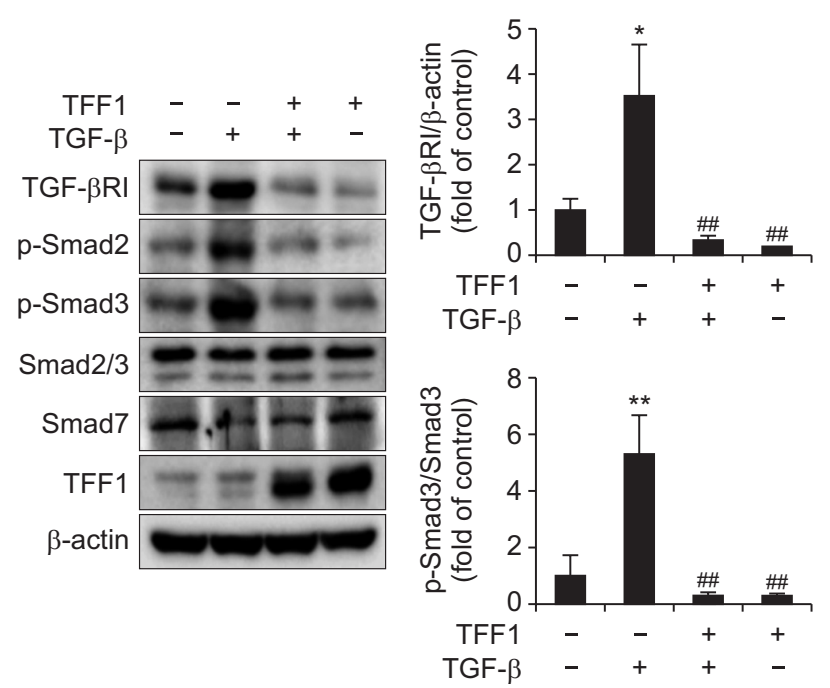

TGF- $\beta$ signal occurs through two types of receptors: I and II complexes [24]. Of these, the receptor that plays an important role in EMT induction has been reported as type I, and therefore, inhibition of TGF- $\beta$ RI in cells also blocks EMT and increases the manifestation of the epithelial phenotype [25]. It has also been reported that inhibiting the function of TGF$\beta R \mathrm{I}$ suppresses EMT and blocks mesenchymal differentiation in vivo [26]. TGF- $\beta R$ I induces the activation of Smad2 and Smad3 [5]. Smad2/3 is phosphorylated and translocate to the nucleus together with Smad4 to regulate the expression of target genes such as E-cadherin, $\mathrm{N}$-cadherin, Snail, Slug and Twist, thereby increasing EMT [5]. On the other hand, Smad6 and Smad7 inhibit the phosphorylation of Smad2/3 mentioned above [24]. Regarding gastric cancer, treatment with compounds that inhibit TGF- $\beta$ signaling has been reported to prevent EMT and metastasis [27], and also to inhibit metastasis induced by TGF- $\beta$ [28]. It has been reported that the expression of TFF1 is decreased while the expression of TGF- $\beta$ is increased in gastric cancer [29]. However, there are few studies on how TFF1 interacts with TGF- $\beta$. TFF1 has been found to be highly related to the estrogen receptor (ER) [30] and ER activation suppresses the transcriptional activity of TGF- $\beta$ [31]. Therefore, the association between TFF1 and TGF- $\beta$ cannot be excluded. Therefore, more in-depth studies on TFF1 and TGF- $\beta$ are needed.

TFF protein is a small molecule peptide with a trefoil domain, and there are three isoforms: TFF1, TFF2 and TFF3 [32]. TFF1 and TFF3 have a single trefoil domain, and TFF2 has two trefoil domains [32]. It is known that the TFF protein primarily plays a role in healing wounds in the gastrointestinal tract [32]. All TFFs are highly resistant to proteolytic enzymes, so they can survive in the strong acidic conditions of the stomach [33]. In particular, TFF1 is expressed in the mucous cells of the fundus and antrum in the stomach, TFF2 is expressed in the mucosal cervical cells of the fundus and the basal cells of the pylorus, and TFF 3 is expressed in the small

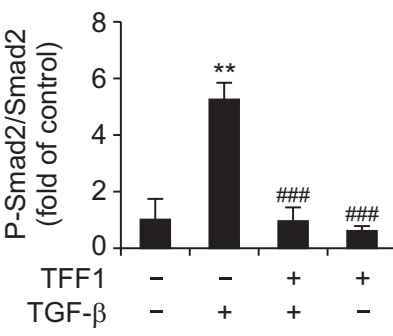

Figure 5. TFF1 inhibits TGF- $\beta$ signaling in AGS cells. AGS cells were transfected with TFF1 and were treated TGF- $\beta$ for 48 hours. Western

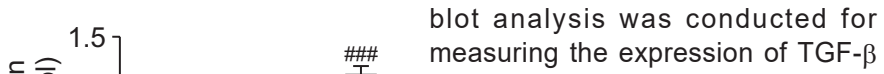
receptor I (TGF- $\beta$ RI), phosphorylation of Smad2 and Smad3, total Smad2/3, and Smad7. TFF1, Trefoil factor 1. ${ }^{*} P$ $<0.05,{ }^{* *} P<0.01$, and ${ }^{* * *} P<0.001$, significantly different compared with control; ${ }^{\#} P<0.01$ and ${ }^{\# \#} P<0.001$, significantly different compared with TGF- $\beta$-treated cells.

and large intestine [32]. TFF1 is generally known to interact with soluble gastric mucosa MUC5AC and is upregulated after mucosal injury, and it has been reported that TFF1 protects the gastric mucosa of transgenic mice with trefoil peptide uptake and TFF1 overexpression [34-37].

TFF1 is a well-known tumor suppressor gene [33]. It has been reported that TFF1 null mice develop adenomas, and about $30 \%$ of these progress to gastric adenocarcinoma [10]. In addition, the protein expression of TFF1 was found to be reduced by about $50 \%$ in the tissues of gastric cancer patients [38], and the mRNA expression of TFF1 was reported to be significantly reduced in human gastric cancer compared to the normal gastric mucosa [39]. Therefore, we thought that inhibition of TFF1 could control gastric cancer based on the fact that the gastric mucosa is protected by TFF1 and that loss of TFF1 cause gastric cancer. In fact, we found that TFF1 did inhibit EMT and metastasis in gastric cancer.

Recently, Yamaguchi et al. [13] confirmed that inhibition of TFF1 suppressed the expression of E-cadherin and increased the expression of Twist and Snail in pancreatic epithelial carcinoma. This suggests that TFF1 can inhibit EMT, consistent with our findings. TFF1 has also been reported as an estrogen-related receptor gene. As Estrogen-related receptor gamma is activated, TFF1 increases, followed by enhancement of mesenchymal-to-epithelial transition, and this led to the inhibition of the growth of breast cancer [40]. On the other hand, a recent study has demonstrated that TFF1 promotes EMT through an auto-induction mechanism in gastric cancer [41]. However, according to the previous studies, TFF1 mutations rather increase the invasion of gastric cancer [33], so it is thought that upregulation of TFF1 plays a positive role in the treatment of gastric cancer.

Studies on TFF1 and its gastric protective effects have been steadily increasing, but TFF-binding molecules have not been identified yet [42]. Recent findings have shown that TFF2 is involved in immune cell recruitment by regulating 
extracellular signal-regulated kinases $1 / 2$ signaling through chemokine receptor (CXCR) $4[42,43]$. It has also been reported that TFF2 affects mucosal therapy through protease-activated receptor 4 [44]. It was also found that TFF3 affects cell migration through CXCR4 and CXCR7 [43], and that TFF3 suppresses the expression of pro-inflammatory cytokines through protease-activated receptor 2 [45]. However, it remains still unknown how TFF1 affects cells. In this study, we confirmed that TFF1 can inhibit TGF- $\beta$ signaling pathway. However, the molecular details how TFF1 inhibits TGF- $\beta$ signaling need to be substantiated by further studies. Through the present study, we speculate that TGF- $\beta$ has potential as a binding molecule for TFF1.

In conclusion, our results show that the overexpression of TFF1 inhibits EMT through regulation of TGF- $\beta$ signaling in gastric cancer cells. In addition, the overexpression of TFF1 also reduced migration and invasiveness of gastric cancer cells as well as the expression of cancer metastasis markers such as MMP-2, MMP-7 and MMP-9. These findings suggest that the assessment of TFF1 status in gastric cancers might be useful during anticancer treatment. Strategies for upregulation of TFF1 levels may influence the effectiveness of anticancer treatment of advanced stage cancers with a high probability of metastasis. Further studies will be necessary to clarify the role of TFF1 and TGF- $\beta$ in the gastric cancer metastasis and EMT.

\section{ACKNOWLEDGMENTS}

This work was supported by the GRRC program of Gyeonggi province (GRRC-201900830002-CHA2019-B01, Production of physiologically active substances) and the Basic Science Research Program through the National Research Foundation (NRF) funded by Ministry of Education (No. 2020R1A6A3A13070372), Republic of Korea.

\section{CONFLICTS OF INTEREST}

No potential conflicts of interest were disclosed.

\section{ORCID}

Da-Young Lee, https://orcid.org/0000-0001-7655-6740

Moon-Young Song, https://orcid.org/0000-0001-9311-5146

Eun-Hee Kim, https://orcid.org/0000-0002-8523-0440

\section{REFERENCES}

1. Rawla P, Barsouk A. Epidemiology of gastric cancer: global trends, risk factors and prevention. Prz Gastroenterol 2019; 14:26-38.

2. Jung KW, Won YJ, Hong S, Kong HJ, Lee ES. Prediction of cancer incidence and mortality in Korea, 2020. Cancer Res Treat 2020;52:351-8.
3. Peng Z, Wang CX, Fang EH, Wang GB, Tong Q. Role of epithelial-mesenchymal transition in gastric cancer initiation and progression. World J Gastroenterol 2014;20:5403-10.

4. Hu WQ, Wang LW, Yuan JP, Yan SG, Li JD, Zhao HL, et al. High expression of transform growth factor beta 1 in gastric cancer confers worse outcome: results of a cohort study on 184 patients. Hepatogastroenterology 2014;61:245-50.

5. Xu J, Lamouille S, Derynck R. TGF-beta-induced epithelial to mesenchymal transition. Cell Res 2009;19:156-72.

6. Miettinen PJ, Ebner R, Lopez AR, Derynck R. TGF-beta induced transdifferentiation of mammary epithelial cells to mesenchymal cells: involvement of type I receptors. J Cell Biol 1994;127(6 Pt 2):2021-36.

7. Leung WK, Yu J, Chan FK, To KF, Chan MW, Ebert MP, et al. Expression of trefoil peptides (TFF1, TFF2, and TFF3) in gastric carcinomas, intestinal metaplasia, and non-neoplastic gastric tissues. J Pathol 2002;197:582-8.

8. Longman RJ, Douthwaite J, Sylvester PA, Poulsom R, Corfield AP, Thomas MG, et al. Coordinated localisation of mucins and trefoil peptides in the ulcer associated cell lineage and the gastrointestinal mucosa. Gut 2000;47:792-800.

9. May FE, Westley BR. Trefoil proteins: their role in normal and malignant cells. J Pathol 1997;183:4-7.

10. Lefebvre O, Chenard MP, Masson R, Linares J, Dierich A, LeMeur $M$, et al. Gastric mucosa abnormalities and tumorigenesis in mice lacking the pS2 trefoil protein. Science 1996;274:259-62.

11. Calnan DP, Westley BR, May FE, Floyd DN, Marchbank T, Playford RJ. The trefoil peptide TFF1 inhibits the growth of the human gastric adenocarcinoma cell line AGS. J Pathol 1999;188:312-7.

12. Park WS, Oh RR, Park JY, Lee JH, Shin MS, Kim HS, et al. Somatic mutations of the trefoil factor family 1 gene in gastric cancer. Gastroenterology 2000;119:691-8.

13. Yamaguchi J, Yokoyama Y, Kokuryo T, Ebata T, Enomoto A, Nagino M. Trefoil factor 1 inhibits epithelial-mesenchymal transition of pancreatic intraepithelial neoplasm. J Clin Invest 2018;128:3619-29.

14. Soutto M, Chen Z, Katsha AM, Romero-Gallo J, Krishna US, Piazuelo MB, et al. Trefoil factor 1 expression suppresses Helicobacter pylori-induced inflammation in gastric carcinogenesis. Cancer 2015;121:4348-58.

15. Song $X, X$ in N, Wang W, Zhao C. Wnt/ $/$-catenin, an oncogenic pathway targeted by $\mathrm{H}$. pylori in gastric carcinogenesis. Oncotarget 2015;6:35579-88.

16. Ge Y, Zhang J, Cao J, Wu Q, Sun L, Guo L, et al. TFF1 inhibits proliferation and induces apoptosis of gastric cancer cells in vitro. Bosn J Basic Med Sci 2012;12:74-81.

17. Aiello NM, Kang Y. Context-dependent EMT programs in cancer metastasis. J Exp Med 2019;216:1016-26.

18. Zhang $X$, Zhang $P$, Shao M, Zang $X$, Zhang J, Mao F, et al. SALL4 activates TGF- $\beta / S M A D$ signaling pathway to induce EMT and promote gastric cancer metastasis. Cancer Manag Res 2018;10:4459-70.

19. Chaffer CL, Weinberg RA. A perspective on cancer cell 
metastasis. Science 2011;331:1559-64.

20. Huang L, Wu RL, Xu AM. Epithelial-mesenchymal transition in gastric cancer. Am J Transl Res 2015;7:2141-58.

21. Thiery JP, Sleeman JP. Complex networks orchestrate epithelialmesenchymal transitions. Nat Rev Mol Cell Biol 2006;7:131-42.

22. Li R, Liang J, Ni S, Zhou T, Qing X, Li H, et al. A mesenchymalto-epithelial transition initiates and is required for the nuclear reprogramming of mouse fibroblasts. Cell Stem Cell 2010;7:5163.

23. De Craene B, Berx G. Regulatory networks defining EMT during cancer initiation and progression. Nat Rev Cancer 2013;13:97110.

24. Feng $\mathrm{XH}$, Derynck R. Specificity and versatility in TGF-beta signaling through Smads. Annu Rev Cell Dev Biol 2005;21:65993.

25. Lamouille S, Derynck R. Cell size and invasion in TGF-betainduced epithelial to mesenchymal transition is regulated by activation of the mTOR pathway. J Cell Biol 2007;178:437-51.

26. Mercado-Pimentel ME, Hubbard AD, Runyan RB. Endoglin and Alk5 regulate epithelial-mesenchymal transformation during cardiac valve formation. Dev Biol 2007;304:420-32.

27. He Z, Dong W, Li Q, Qin C, Li Y. Sauchinone prevents TGF- $\beta$ induced EMT and metastasis in gastric cancer cells. Biomed Pharmacother 2018;101:355-61.

28. Sarkar A, Das S, Rahaman A, Das Talukdar A, Bhattacharjee S, Mandal DP. Eugenol and capsaicin exhibit anti-metastatic activity via modulating TGF- $\beta$ signaling in gastric carcinoma. Food Funct 2020;11:9020-34.

29. Morita K, Fujimori T, Ono Y, Hiraishi H, Yoshiura K, Shimada $\mathrm{T}$, et al. Identification of the prelinitis condition in gastric cancer and analysis of TGF-beta, TGF-beta RII and pS2 expression. Pathobiology 2001;69:321-8.

30. Rio MC, Bellocq JP, Gairard B, Rasmussen UB, Krust A, Koehl $C$, et al. Specific expression of the pS2 gene in subclasses of breast cancers in comparison with expression of the estrogen and progesterone receptors and the oncogene ERBB2. Proc Natl Acad Sci USA 1987;84:9243-7.

31. Matsuda T, Yamamoto T, Muraguchi A, Saatcioglu F. Cross-talk between transforming growth factor-beta and estrogen receptor signaling through Smad3. J Biol Chem 2001;276:42908-14.

32. Katoh $M$. Trefoil factors and human gastric cancer (review). Int $J$ Mol Med 2003;12:3-9.

33. Yio X, Diamond M, Zhang JY, Weinstein H, Wang LH, Werther L, et al. Trefoil factor family-1 mutations enhance gastric cancer cell invasion through distinct signaling pathways. Gastroenterology
2006;130:1696-706.

34. Ruchaud-Sparagano MH, Westley BR, May FE. The trefoil protein TFF1 is bound to MUC5AC in human gastric mucosa. Cell Mol Life Sci 2004;61:1946-54.

35. Wright NA, Poulsom R, Stamp GW, Hall PA, Jeffery RE, Longcroft JM, et al. Epidermal growth factor (EGF/URO) induces expression of regulatory peptides in damaged human gastrointestinal tissues. J Pathol 1990;162:279-84.

36. Babyatsky MW, deBeaumont M, Thim L, Podolsky DK. Oral trefoil peptides protect against ethanol- and indomethacininduced gastric injury in rats. Gastroenterology 1996;110:489-97.

37. Playford RJ, Marchbank T, Goodlad RA, Chinery RA, Poulsom R, Hanby AM. Transgenic mice that overexpress the human trefoil peptide pS2 have an increased resistance to intestinal damage. Proc Natl Acad Sci USA 1996;93:2137-42.

38. Carvalho R, Kayademir T, Soares P, Canedo P, Sousa S, Oliveira $\mathrm{C}$, et al. Loss of heterozygosity and promoter methylation, but not mutation, may underlie loss of TFF1 in gastric carcinoma. Lab Invest 2002;82:1319-26.

39. Beckler AD, Roche JK, Harper JC, Petroni G, Frierson HF Jr, Moskaluk CA, et al. Decreased abundance of trefoil factor 1 transcript in the majority of gastric carcinomas. Cancer 2003;98:2184-91.

40. Tiraby C, Hazen BC, Gantner ML, Kralli A. Estrogen-related receptor gamma promotes mesenchymal-to-epithelial transition and suppresses breast tumor growth. Cancer Res 2011;71:251828.

41. Romano E, Vllahu M, Bizzarro V, Belvedere R, Esposito R, Petrella A, et al. TFF1 promotes EMT-like changes through an auto-induction mechanism. Int J Mol Sci 2018;19:2018.

42. Hoffmann W. Trefoil factor family (TFF) peptides and chemokine receptors: a promising relationship. J Med Chem 2009;52:650510.

43. Dieckow J, Brandt W, Hattermann K, Schob S, Schulze U, Mentlein R, et al. CXCR4 and CXCR7 mediate TFF3-induced cell migration independently from the ERK1/2 signaling pathway. Invest Ophthalmol Vis Sci 2016;57:56-65.

44. Zhang Y, Yu G, Wang Y, Xiang Y, Gao Q, Jiang P, et al. Activation of protease-activated receptor (PAR) 1 by frog trefoil factor (TFF) 2 and PAR4 by human TFF2. Cell Mol Life Sci 2011;68:3771-80.

45. Barrera GJ, Tortolero GS. Trefoil factor 3 (TFF3) from human breast milk activates PAR-2 receptors, of the intestinal epithelial cells HT-29, regulating cytokines and defensins. Bratisl Lek Listy 2016;117:332-9. 